\title{
THE SEIBERG-WITTEN EQUATIONS FOR FAMILIES AND DIFFEOMORPHISMS OF 4-MANIFOLDS *
}

\author{
NOBUHIRO NAKAMURA ${ }^{\dagger}$
}

1. Introduction. In this paper, we investigate the Seiberg-Witten equations for families, and get some information about diffeomorphisms of 4-manifolds. The Seiberg-Witten equations for families have been investigated by several authors [4], [6], [9], [12]. Our concern is related to reducible solutions.

Reducible solutions of the Seiberg-Witten equations play an important role in a proof of Donaldson's theorem [2] for a closed 4-manifold of $b^{+}=0$ (See e.g. [10]).

On the other hand, in the case when $b^{+}=1$, the Seiberg-Witten invariants can not be diffeomorphism invariants. The phenomenon is also related to the existence of reducible solutions. The fact is used by [7] to give a proof of the Thom conjecture.

The main topic of this paper is a generalization of the above two situations. We concentrate on the case of just $b^{+}$-dimensional family of a 4-manifold.

As applications, by considering the families over $S^{1}$ and torus, we prove the following results about diffeomorphisms of non-spin manifolds of $b^{+}=1,2$ with even intersection forms.

THEOREM 1.1. Let $X$ be a closed 4-manifold of $b_{1}=0$ whose intersection form is of the form $-E_{8} \oplus 2 H$, where $H$ is the hyperbolic quadratic form. Then there do not exist orientation-preserving commutative diffeomorphisms $f$ and $g$ which have the following two properties:

(1) $f$ and $g$ preserve an integral lift of $w_{2}(X)$ which is in the torsion part of $H^{2}(X ; \mathbb{Z})$.

(2) Decompose $H^{2}(X ; \mathbb{Z}) /$ Tor into the direct sum $-E_{8} \oplus H \oplus H$. Let $f^{*}, g^{*}$ be automorphisms on $H^{2}(X ; \mathbb{Z}) /$ Tor induced from $f, g$. Then $f^{*}$ and $g^{*}$ are of the forms,

$$
\begin{aligned}
& f^{*}=F \oplus(-1) \oplus(+1), \\
& g^{*}=G \oplus(+1) \oplus(-1),
\end{aligned}
$$

where $F, G$ are commutative matrices which preserve $E_{8}$.

C. Bohr constructed many examples of $X$ which satisfy the assumption of Theorem $1.1[1]$.

THEOREM 1.2. Let $X$ be a closed 4-manifold of $b_{1}=0$ whose intersection form is of the form $-E_{8} \oplus H$. Let $f: X \rightarrow X$ be an orientation-preserving diffeomorphism of $X$, and suppose that $f$ preserves an integral lift of $w_{2}(X)$ which is in the torsion part of $H^{2}(X ; \mathbb{Z})$. Then $f$ preserves the orientation of $H^{+}(X ; \mathbb{R})$.

Particularly, in the case of the Enriques surface, we have the following.

CoRollary 1.3. An orientation-preserving diffeomorphism of the Enriques surface preserves the orientation of $H^{+}(X ; \mathbb{R})$.

A similar result for $K 3$ surface is well-known [3].

\footnotetext{
*Received July 17, 2002; accepted for publication February 27, 2003.

†Research Institute for Mathematical Sciences, Kyoto University, Kyoto, 606-8502, Japan (nakamura@kurims.kyoto-u.ac.jp).
} 
Let us explain the central part of the argument.

Suppose that we have a family of 4-manifold parametrized by $B$. Precise formulation is as follows. Let $B$ be a $d$-dimensional closed manifold, and $X$ be an oriented closed 4-manifold of $b^{+}=d$. Furthermore, we assume that $b_{1}$ of $X$ is 0 for simplicity. Let $\mathbb{X}$ be a smooth fiber bundle over $B$ with fiber $X$. On such a family, we consider the Seiberg-Witten equations and the union of the moduli spaces of solutions for all the parameters. Let us denote the union by $\mathcal{M}$. Then, even if we choose perturbation generically, we can not avoid reducibles in general. When $b_{1}=0, \mathcal{M}$ contains some reducibles as singular points. Let $n$ be the dimension of $\mathcal{M}$. (In this situation, $n$ is odd.) Then we can see that the neighborhood of a reducible becomes a cone of $\mathbb{C P}^{\frac{n-1}{2}}$. Removing these cones from $\mathcal{M}$, we get a $n$-dimensional compact manifold whose boundary consists of several $\mathbb{C P}^{\frac{n-1}{2}}$,s. In the unoriented cobordism group of $4 k$-dimension, $\mathbb{C P}^{2 k}$ is a non-trivial element. Hence, if $n$ is equal to 1 modulo 4 , then the number of components of boundary, that is, the number of reducibles must be even (Theorem 2.4).

The fact that the number of reducibles must be even will imply a constraint on the topology of smooth $X$-bundle. Applying this to the case when $B$ is $S^{1}$ or torus, we can get Theorem 1.1 and Theorem 1.2.

Acknowledgements. The author would like to thank M. Furuta for invaluable discussions.

2. The Seiberg-Witten equations for a family. Let $X$ be an oriented closed 4-manifold. Let $b_{i}$ denote the $i$-th Betti number of $X$ and $b^{+}$denote the dimension of a maximal subspace, $H^{+}(X ; \mathbb{R})$ in $H^{2}(X ; \mathbb{R})$, where the intersection form is positive definite. In this paper, we suppose that $b_{1}$ of $X$ is 0 .

Let $B$ be another oriented closed $d$-dimensional manifold, and $\mathbb{X}$ be a smooth fiber bundle over $B$ with fiber $X$. Let us denote the tangent bundle along the fiber by $T(\mathbb{X} / B)$ and the bundle of $i$-forms by $\Omega^{i}(\mathbb{X} / B)$. By choosing a metric on $T(\mathbb{X} / B)$, we get a principal $\mathrm{SO}(4)$ bundle $F r$ of frames.

A Spin ${ }^{c}$-structure $\mathbb{P}$ on $T(\mathbb{X} / B)$ is an equivalence class of lifts of $\mathrm{SO}(4)$ bundle $F r$ to a $\operatorname{Spin}^{c}(4)$ bundle $\mathbb{P}$. (We abuse the notations for a $\operatorname{Spin}^{c}$-structure and a $\operatorname{Spin}^{c}(4)$ bundle.)

Suppose that a $\operatorname{Spin}^{c}$-structure $\mathbb{P}$ is given. Then, by restricting this $\operatorname{Spin}^{c}$ structure to a fiber $X_{b}$ at $b \in B$ of $\mathbb{X}$, we have a Spin ${ }^{c}$-structure $\mathbb{P}_{b}$ on $X_{b}$. (For any object on the total space $\mathbb{X}$, the object with subscript $b$ will denote the restriction to the fiber $X_{b}$.)

Conversely, suppose that a $\operatorname{Spin}^{c}$-structure $P$ on $X$ is given. We want to construct a Spin ${ }^{c}$-structure on $T(\mathbb{X} / B)$ from $P$. In general, however, this is not possible. In what situation can we do that? The following is an answer.

Proposition 2.1. Suppose that $C \in H^{2}(X ; \mathbb{Z})$ and a fiber bundle $\mathbb{X} \rightarrow B$ with fiber $X$ satisfy the following conditions:

(1) The class $C$ is an integral lift of $w_{2}(X)$ and $C \in H^{2}(X ; \mathbb{Z})^{\pi_{1}(B)}$.

(2) $H^{3}(B ; \mathbb{Z})=0$.

(3) The map of $\bmod 2$ reduction $r_{1}: H^{2}(B ; \mathbb{Z}) \rightarrow H^{2}\left(B ; \mathbb{Z}_{2}\right)$ is surjective.

Then there is a Spin ${ }^{c}$-structure $\mathbb{P}$ on $T(\mathbb{X} / B)$ such that $c_{1}\left(\left.\operatorname{det} \mathbb{P}\right|_{X}\right)=C$.

Proof. By considering the spectral sequence for the fiber bundle $\mathbb{X} \rightarrow B$, we can 
get the following commutative diagram.

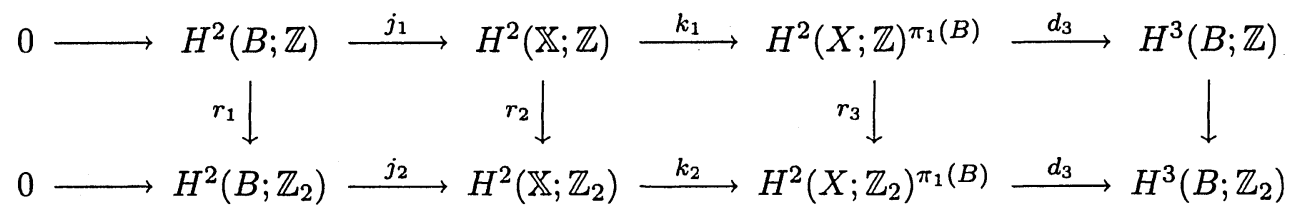

(The horizontal arrows are exact. The maps $d_{3}$ are transgression. The vertical arrows are mod 2 reduction maps.) First note that $w_{2}(X)=k_{2}\left(w_{2}(T(\mathbb{X} / B))\right)=r_{3}(C)$. By the assumption (2), there is a lift $\tilde{C} \in H^{2}(\mathbb{X} ; \mathbb{Z})$ of $C \in H^{2}(X ; \mathbb{Z})^{\pi_{1}(B)}$ via $k_{1}$. The image $C^{\prime} \in H^{2}\left(\mathbb{X} ; \mathbb{Z}_{2}\right)$ of $\tilde{C}$ by $r_{2}$ does not necessarily coincide with $w_{2}(T(\mathbb{X} / B)$ ). But, by the commutativity of the diagram, the image of $C^{\prime}-w_{2}(T(\mathbb{X} / B))$ by $k_{2}$ is 0 . Hence there is a lift $\alpha \in H^{2}(B ; \mathbb{Z})$ of $C^{\prime}-w_{2}(T(\mathbb{X} / B))$ via $j_{2}$. By the assumption (3), we have a lift $\tilde{\alpha}$ of $\alpha$ via $r_{1}$. Then $\tilde{C}-j_{1}(\tilde{\alpha})$ is a lift of $w_{2}(T(\mathbb{X} / B))$. This proves the existence of required $\operatorname{Spin}^{c}$-structure. $\square$

Next we want to consider the Seiberg-Witten equations for a family. Fix a class $C \in H^{2}(X ; \mathbb{Z})$ as in Proposition 2.1, and fix a Spin ${ }^{c}$-structure $\mathbb{P}$ associated to it. Let $S^{+}$denote the positive spinor bundle and $L$ denote $\operatorname{det}\left(S^{+}\right)$. The bundle $L$ is viewed as a family of $\mathrm{U}(1)$ bundles over $B: L=\coprod_{b \in B} L_{b}$. Let us denote the space of $\mathrm{U}(1)$ connections on $L_{b}$ by $\mathcal{A}\left(L_{b}\right)$.

Let us define the bundle $\Pi \rightarrow B$ of parameters as follows:

$$
\Pi=\left\{\left(g_{b}, \mu_{b}\right) \in \operatorname{Met}\left(X_{b}\right) \times \Omega^{2}\left(X_{b}\right) \mid * g_{b} \mu_{b}=\mu_{b}\right\},
$$

where $\operatorname{Met}\left(X_{b}\right)$ denote the space of metrics on $X_{b}$.

When we choose a section $\eta: B \rightarrow \Pi$, we get the Seiberg-Witten equations on $\mathbb{P}$ for a family $\left\{\left(A_{b}, \psi_{b}\right)\right\}_{b \in B} \in \coprod_{b \in B} \mathcal{A}\left(L_{b}\right) \times \Gamma\left(S_{b}^{+}\right)$:

$$
\left\{\begin{aligned}
D_{A_{b}} \psi_{b} & =0 \\
F_{A_{b}}^{+g_{b}} & =\left(\psi_{b} \otimes \psi_{b}^{*}\right)_{0}+i \mu_{b},
\end{aligned}\right.
$$

where $D_{A_{b}}$ denotes the Dirac operator defined by a connection $A_{b}$ and the Levi-Civita connection of metric $g_{b},\left(\psi_{b} \otimes \psi_{b}^{*}\right)_{0}$ denotes the trace-free part of $\psi_{b} \otimes \psi_{b}^{*}$ interpreted as an endomorphism of $S^{+}$(and this endomorphism is identified with an imaginaryvalued self-dual 2 -form via the Clifford multiplication).

The action of gauge transformation group $\mathcal{G}_{b}=\operatorname{Map}\left(X_{b} ; \mathrm{U}(1)\right)$ is as follows: for $u_{b} \in \mathcal{G}_{b}, u_{b}\left(A_{b}, \psi_{b}\right)=\left(A_{b}-u_{b}^{-1} d u_{b}, u_{b} \psi_{b}\right)$. The group $\mathcal{G}_{b}$ acts freely at $\left(A_{b}, \psi_{b}\right)$ where $\psi_{b}$ is not identically 0 .

A solution to the Seiberg-Witten equations with the property $\psi_{b} \equiv 0$ is called a reducible solution. The subspace $\mathcal{W}$ of $\Pi$ where reducible solutions appear is called the wall. The wall $\mathcal{W}$ is characterized as

$$
\mathcal{W}=\left\{\left(g_{b}, \mu_{b}\right) \mid 2 \pi C-\mu_{b} \text { is } g_{b} \text {-anti-self-dual }\right\},
$$

where $C$ is considered as a harmonic 2 -form. The wall $\mathcal{W}$ has codimension $b^{+}$in $\Pi$.

The moduli space of the solutions to the Seiberg-Witten equations for a family is given as

$$
\mathcal{M}_{\eta}(\mathbb{X}, \mathbb{P})=\coprod_{b \in B}\{\text { solutions to }(2.2)\} / \mathcal{G}_{b}
$$


The virtual dimension $d(C)$ of the moduli space is

$$
d(C)=\frac{1}{4}\left(C^{2}-\operatorname{sign}(X)\right)-1-b^{+}+d .
$$

(We assume that $b_{1}=0$.)

If we choose $\eta$ generically, then $\mathcal{M}_{\eta}(\mathbb{X}, \mathbb{P})$ becomes a $d(C)$-dimensional smooth manifold outside reducibles.

When $\eta$ and $\mathcal{W}$ intersect, reducible solutions appear, and they form a $b_{1}$ dimensional torus $H^{1}(X ; \mathbb{R}) / H^{1}(X ; \mathbb{Z})$ in the moduli space. Therefore, if $b_{1}=0$, each time $\eta$ crosses $\mathcal{W}$, one reducible point appears in the moduli space.

To see the intersection of $\eta$ and $\mathcal{W}$, we introduce a finite dimensional vector bundle $H_{\eta}^{+}$over $B$ as follows: For each $b \in B$, let $H_{g_{b}}^{+} \subset \Omega^{2}\left(X_{b}\right)$ be the space of $g_{b}$-self-dual harmonic 2-forms. The spaces $H_{g_{b}}^{+}$form a $b^{+}$-dimensional subbundle $H_{\eta}^{+} \rightarrow B$ of $\Omega^{2}(\mathbb{X} / B) \rightarrow B$

Let us define the section $s_{\eta}$ of $H_{\eta}^{+}$by $s_{\eta}(b)=2 \pi C-h\left(\mu_{b}\right) \cup$, where $h\left(\mu_{b}\right)$ denote the harmonic part of $\mu_{b}$, and $H_{\eta}^{+}$is identified with its dual bundle via the cup product.

Now let us consider the situation that the dimension $d$ of $B$ is equal to $b^{+}$of $X$. Then $d(C)$ in (2.3) becomes an odd number.

Suppose further that $\eta$ is generic and intersects $\mathcal{W}$ transversally. (Then the section $s_{\eta}$ of $H_{\eta}^{+}$intersects the zero section transversally.) Note that the intersection of $\eta$ and $\mathcal{W}$ consists of discrete points of finite number. Note also that there is one-to-one correspondence between intersection points of $\eta$ and $\mathcal{W}$, and zeros of $s_{\eta}$. Hence, when $b_{1}=0$, there is one-to-one correspondence between reducibles in the moduli space and zeros of $s_{\eta}$.

The next theorem will imply a constraint on the topology of smooth $X$-bundle, as we can see in Section 3.

TheOREM 2.4. If $d(C)>0$ and $d(C) \equiv 1(\bmod 4)$, the number of zeros of the section $s_{\eta}$ is even.

Proof. Outside reducibles, the moduli space $\mathcal{M}(\mathbb{X}, \mathbb{P})$ forms a $d(C)$-dimensional smooth manifold which may not be orientable. In the neighborhoods of reducibles, if necessary, perturb the Dirac operator in (2.2). The Kuranishi model is given by a $\mathrm{U}(1)$-equivariant map,

$$
\Phi: \mathbb{C}^{m+\frac{1}{8}\left(C^{2}-\operatorname{sign}(X)\right)} \oplus \mathbb{R}^{n} \rightarrow \mathbb{C}^{m} \oplus \mathbb{R}^{n+b^{+}-d},
$$

for some $m, n \in \mathbb{N}$. Since we assume that $b^{+}-d=0$, the neighborhood of a reducible is homeomorphic to the cone of $\mathbb{C P} \frac{d(C)-1}{2}$. (The case of 1-dimensional family is explained in [11]. The argument there is directly applied to our case.) Remove the cones from $\mathcal{M}(\mathbb{X}, \mathbb{P})$. Then we get a $d(C)$-dimensional compact manifold whose boundary consists of several $\mathbb{C} P^{\frac{d(C)-1}{2}}$ 's. In the unoriented cobordism group of $4 k$-dimension, $\mathbb{C P}^{2 k}$ is a non-trivial element. Hence, under the assumption of theorem, the number of components of boundary must be even. Noticing that the number of components of boundary is equal to the number of reducibles, and a reducible corresponds to a zero of $s_{\eta}$, we see the theorem.

REMARK 2.5. In the case when $H_{\eta}^{+} \rightarrow B$ is orientable, we can refine Theorem 2.4 as follows: If $d(C)>0$ and $d(C) \equiv 1(\bmod 4)$, the signed count of zeros of $s_{\eta}$ according to its orientations is 0 . 
REMARK 2.6. When $d(C)>0$ and $d(C) \equiv 3(\bmod 4)$, the assertion of Theorem 2.4 still holds in some cases. Consider the space $\mathcal{B}_{b}^{*}=\mathcal{A}\left(L_{b}\right) \times\left(\Gamma\left(S_{b}^{+}\right) \backslash\left\{\psi_{b} \equiv\right.\right.$ $0\}) / \mathcal{G}_{b}$. When $b_{1}=0$, it is known that $\mathcal{B}_{b}^{*}$ has the same homotopy type of $\mathbb{C P}^{\infty}$ which has non-zero second cohomology class $U$. If $U$ has a lift $\tilde{U}$ in the cohomology of the family $\coprod_{b \in B} \mathcal{B}_{b}^{*}$, we can deduce the assertion of Theorem 2.4 by evaluating the fundamental class of $\mathbb{C P} \frac{d(C)-1}{2}$ of the moduli's boundary by $\tilde{U}^{\frac{d(C)-1}{2}}$.

3. Applications of Theorem 2.4. In this section, we prove theorems in the introduction as applications of Theorem 2.4.

3.1. The case when $d=b^{+}=1$. In this part, we deal with a manifold $X$ of $b_{1}=0$ whose intersection form is of the form $-E_{8} \oplus H$. For example, let $X$ be the Enriques surface. As for the topology of the Enriques surface, see e.g. [5].

Note that, when a 4-manifold $X$ has even intersection form, $w_{2}(X)$ has an integral lift which is in the torsion part of $H^{2}(X ; \mathbb{Z})$. (This can be seen easily from Wu's formula and the universal coefficient theorem. See e.g.[8].)

Let us prove Theorem 1.2 and Corollary 1.3.

Proof of Theorem 1.2. Let $C$ be an integral lift of $w_{2}(X)$ as in Theorem 1.2. Let us consider the mapping cylinder $\mathbb{X}=X \times[0,1] / f \rightarrow S^{1}$. In this case, $B=S^{1}$ and $f$ preserves the class $C$. Hence, by Proposition 2.1, there is a $\operatorname{Spin}^{c}$-structure $\mathbb{P}$ with the property $c_{1}(\operatorname{det} \mathbb{P} \mid X)=C$. Note that $d(C)=1$. By Theorem 2.4, we see that the number of zeros of the section $s_{\eta}$ is even.

Suppose now that the diffeomorphism $f$ reverse the orientation of $H^{+}(X ; \mathbb{R})$. Then $H_{\eta}^{+}$is the non-trivial $\mathbb{R}$-bundle over $S^{1}$ which can not have a transversal section whose number of zeros is even. This is contradiction. $\square$

Proof of Corollary 1.3. When $X$ is the Enriques surface, the torsion part of $H^{2}(X ; \mathbb{Z})$ is $\mathbb{Z}_{2}$. Hence, the assumption concerning $w_{2}(X)$ is automatically satisfied.

REMARK 3.1. As for the case of this part, the fiberwise dimension of the moduli space is 0 . In this case, one could prove results of this type by ordinary wall-crossing argument.

3.2. The case when $d=b^{+}=2$. In this part, we deal with a manifold $X$ of $b_{1}=0$ whose intersection form is of the form $-E_{8} \oplus 2 H$. For example, let $X$ be the connected sum of the Enriques surface with $S^{2} \times S^{2}$. C. Bohr constructs examples of $\pi_{1}(X)=\mathbb{Z}_{2 k}$ ( $k$ is a positive integer $)$ in Example 2 of [1].

Let us prove Theorem 1.1.

Proof of Theorem 1.1. Suppose that there exist commutative diffeomomorphism $f$ and $g$ which satisfy the properties (1) and (2) in the theorem. Let $C$ be an integral lift of $w_{2}(X)$ as in (1). Let us consider the following $X$-bundle $\mathbb{X}$ over $T^{2}$ :

$$
\mathbb{X}=X \times[0,1] \times[0,1] /\left\{\begin{array}{l}
f(0, t) \sim f(1, t) \\
g(s, 0) \sim g(s, 1)
\end{array}\right\} .
$$

In this case, $B=T^{2}$ and $f$ and $g$ preserve the class $C$. Hence, by Proposition 2.1, there is a $\operatorname{Spin}^{c}$-structure $\mathbb{P}$ with the property $c_{1}(\operatorname{det} \mathbb{P} \mid X)=C$. Note that $d(C)=1$. By Theorem 2.4, we see that the number of zeros of the section $s_{\eta}$ is even.

On the other hand, by the property (2), the bundle $H_{\eta}^{+}$is of the form

$$
\pi_{1}^{*} E \oplus \pi_{2}^{*} E \rightarrow S^{1} \times S^{1},
$$


where $E$ be the non-trivial $\mathbb{R}$-bundle over $S^{1}$, and $\pi_{i}: S^{1} \times S^{1} \rightarrow S^{1}$ be the $i$-th projection $(i=1,2)$. Hence, $w_{2}\left(H_{\eta}^{+}\right)$is non-zero, and this implies contradiction.

REMARK 3.2. In this case, the fiberwise dimension of the moduli space is -1 .

In Theorem 1.1, suppose that $H_{1}(X ; \mathbb{Z})=\mathbb{Z}_{2 k}$ ( $k$ is odd). Then, for all diffeomorphisms, the assumption (1) is satisfied. (Take the element of order 2 as the integral lift of $w_{2}(X)$.) Hence the following holds.

CoROllaRY 3.3. Let $X$ be a manifold whose intersection form is of the form $-E_{8} \oplus 2 H$ and suppose $H_{1}(X ; \mathbb{Z})=\mathbb{Z}_{2 k}$ ( $k$ is odd). There do not exist orientationpreserving commutative diffeomorphisms $f, g$ of $X$ which has the property (2) in Theorem 1.1.

REMARK 3.4. We can formulate similar results in the case when $d=b^{+} \geq 3$. However, these are rather complicated.

\section{REFERENCES}

[1] C. BoHR, Immersed projective planes, Arf invariants and even 4-manifolds, preprint, math.GT/0201163.

[2] S. K. Donaldson, An application of gauge theory to four-dimensional topology, J. Diff. Geom., 18 (1983), pp. 141-168.

[3] S. K. Donaldson, P. B. Kronheimer, The Geometry of Four-Manifold, Oxford, 1990.

[4] M. Furuta, Stable homotopy version of Seiberg-Witten invariant, preprint.

[5] N. HABEGGER, Une variété de dimension 4 avec forme d'intersection paire et signature -8 , Comment. Math. Helvetici, 57 (1982), pp. 22-24.

[6] P. B. KRONHEIMER, Some nontrivial families of symplectic structures, preprint.

[7] P. B. Kronheimer, T. S. MrowkA, The genus of embedded surfaces in the projective plane, Math. Res. Letters, 1 (1994), pp. 797-808.

[8] R. LEE, T. LI, Intersection forms of non-spin four manifolds, Math. Ann., 319 (2001), pp. $311-318$.

[9] T. LI, A. LiU, Family Seiberg-Witten invariants and wall crossing formulas, Comm. Analysis and Geometry, 9:4 (2001), pp. 777-823.

[10] J. D. MOORE, Lectures on Seiberg-Witten invariants, LNM 1629, Springer, 1996.

[11] J. W. MORGAN, The Seiberg-Witten equations and application to the topology of smooth fourmanifolds, Mathematical Notes, Princeton Univ. Press, 1996.

[12] D. Ruberman, Positive scaler curvature, diffeomorphisms and the Seiberg-Witteninvariants, Geom. Topol., 5 (2001), pp. 895-924. 